\title{
Observational study of the effects of age, diabetes mellitus, cirrhosis and chronic kidney disease on sublingual microvascular flow
}

\author{
Toby Reynolds ${ }^{1}$, Amanda Vivian-Smith', Shaman Jhanji ${ }^{2}$ and Rupert M Pearse ${ }^{3^{*}}$
}

\begin{abstract}
Background: Sidestream dark field (SDF) imaging has been used to demonstrate microcirculatory abnormalities in a variety of critical illnesses. The microcirculation is also affected by advancing age and chronic comorbidities. However, the effect of these conditions on SDF microcirculatory parameters has not been well described.

Methods: SDF images were obtained from five groups of 20 participants: healthy volunteers under the age of 25 , healthy volunteers over the age of 55, and clinic patients over the age of 55 with one of diabetes mellitus, cirrhosis and stage 5 chronic kidney disease. Microcirculatory parameters between the groups were then compared for significance using analysis of variance for parametric and the Kruskal-Wallis test for non-parametric data.

Results: Median microvascular flow index was 2.85 (interquartile range 2.75 to 3.0 ) for participants aged $<25,2.81$ ( 2.66 to 2.97 ) for those aged $>55,2.88$ (2.75 to 3.0) for those with diabetes mellitus, 3.0 (2.83 to 3.0) for those with cirrhosis and 3.0 (2.78 to 3.0) for those with chronic kidney disease ( $P$ for difference between groups $=0.14$ ). Similarly, there were no significant differences in the proportion of perfused vessels and perfused vessel density between the groups.
\end{abstract}

Conclusions: Older age, diabetes, and chronic kidney and liver disease need not be considered confounding factors for comparison of SDF microcirculatory parameters in the critically ill.

Keywords: Microvascular flow, Sidestream dark field imaging, Age, Diabetes mellitus, Cirrhosis, Chronic kidney disease

\section{Background}

Critical illness is often accompanied by abnormalities of the microcirculation [1]. Persistent alterations such as heterogeneity of flow and microvascular shunting can lead to tissue hypoxia and are associated with organ failure and death [2-4], while impaired microvascular flow in the perioperative period is associated with the development of complications [5]. Improving systemic measures of perfusion can be accompanied by improved microcirculatory parameters [6] but the relationship between the two is not always straightforward $[7,8]$, including during vasopressor treatment $[9,10]$. Monitoring the microcirculation may thus help evaluate an individual patient's tissue perfusion $[7,11]$.

\footnotetext{
*Correspondence: r.pearse@qmul.ac.uk

${ }^{3}$ Barts and The London School of Medicine and Dentistry, Queen Mary's University of London, Turner Street, London E1 2AD, UK

Full list of author information is available at the end of the article
}

The novel techniques of orthogonal polarisation spectral (OPS) imaging [12], and more recently sidestream dark field (SDF) imaging [13] allow imaging of the microcirculation in vivo in a way that was previously technically difficult. Research using these techniques has helped characterise the sublingual microcirculation in various acute diseases, and describe the changes in microcirculatory parameters following acute physiological changes or administration of pharmacological therapies. However, the influence of existing chronic conditions on microcirculatory assessments made using SDF and OPS imaging has not been well studied. Advancing age, diabetes mellitus, cirrhosis, and end-stage renal failure are commonly found in critically ill perioperative patients $[14,15]$. All have well recognised associations with an abnormal microvasculature, and altered microvascular flow has been demonstrated using other techniques [16-19]. In order to interpret 
current and future clinical studies of the microcirculation using SDF and OPS imaging, it is essential to determine whether stable patients with advanced age and these chronic diseases exhibit changes in sublingual microvasculature when assessed using these techniques. The objective of this study was to help provide this understanding by evaluating sublingual microvascular flow using SDF imaging in healthy older volunteers and in patients with diabetes, cirrhosis and chronic kidney disease (CKD).

\section{Methods}

\section{Study design}

This was a single centre, observational study based at a university hospital, and was approved by East London and the City Research Ethics Committee (ref. 09/H0704/3). Patients aged over 55 years with one of either diabetes (diagnosed $>2$ years), cirrhosis (confirmed by liver biopsy or expert hepatology opinion) or CKD (stage 5 by estimated glomerular filtration rate, but not on dialysis) were identified from amongst those attending diabetology, hepatology or nephrology outpatient clinics. Healthy volunteers aged either under 25 or over 55 years without medical disease were identified from among hospital staff and their relatives. All participants gave written informed consent. Exclusion criteria were pregnancy, age 18 years and refusal of consent.

\section{Data collection}

Sublingual microvascular flow was evaluated using sidestream dark field imaging with a $\mathrm{x} 5$ objective lens (Microscan, Microvision Medical, Amsterdam, Netherlands) [20]. Image acquisition and subsequent blinded analysis was performed according to published consensus criteria [21]. SDF images were obtained from at least three sublingual areas. Microvascular flow index (MFI) was calculated after dividing each image into four equal quadrants. Quantification of flow was determined using an ordinal scale (0: no flow, 1: intermittent flow, 2: sluggish flow, 3: normal flow) for small $(<20 \mu \mathrm{m})$ and large $(>20 \mu \mathrm{m})$ vessels. MFI is the average score of all quadrants for a given category of vessel size. Vessel density was calculated by inserting a grid of three equidistant horizontal and three equidistant vertical lines over the image. Vessel density is equal to the number of vessels crossing these lines divided by their total length. Flow was then categorised as present, intermittent or absent to calculate the proportion of perfused vessels (PPV) and thus the perfused vessel density (PVD), an estimate of functional capillary density (FCD). Analysis of the videos was performed by a single, blinded observer (TR). Additional data collected for all participants included age, weight, gender, past medical history, current medications, pulse rate, sublingual temperature, heart rate and blood pressure. In diabetic patients the most recent $\mathrm{HbA1c}$ percentage result was recorded. In patients with cirrhosis the current Child-Turcotte-Pugh score was recorded. In patients with renal disease, the creatinine clearance was estimated from plasma creatinine using the four-variable modified diet in renal disease method [22].

\section{Statistical analysis}

Data from a previous study, performed locally, demonstrated a difference in MFI of 0.3 prior to major abdominal surgery in those patients who went on to develop complications after surgery compared to those who did not [5]. Assuming a type I error rate of 5\% (two-tailed) and a type II error rate of $10 \%$ using this previous data, 20 patients would be required in each group to detect a difference in MFI of 0.26 (SD \pm 0.25 ). We therefore aimed to recruit 20 participants in each group. Parametric data are presented as mean (standard deviation, SD) and non-parametric data are presented as median (interquartile range, IQR). Variable distributions were assessed for normality using the Kolmogorov-Smirnov test. The primary outcome measure of this study was a difference in small vessel MFI between the groups. Secondary outcome measures were differences in PPV and PVD for small vessels. Differences between groups were tested using analysis of variance (ANOVA) for parametric data and the Kruskal-Wallis test for non-parametric data. Significance was set at $P<0.05$. Statistical calculations were performed in SPSS (SPSS Inc, Chicago, IL, USA).

\section{Results}

One hundred participants were recruited between September 2010 and July 2011. Adequate video footage was obtained in 98 participants $(20$ aged $<25$ years, 20

Table 1 Baseline participant data

\begin{tabular}{llllll}
\hline & Age $<\mathbf{2 5}$ years & Age $>\mathbf{5 5}$ years & Diabetes mellitus & Cirrhosis & Chronic kidney disease \\
\hline Male & $10(50 \%)$ & $9(45 \%)$ & $10(50 \%)$ & $12(67 \%)$ & $14(70)$ \\
Age (years) & $24(23-25)$ & $61(60-65)$ & $64(62-72)$ & $60(57-66)$ & $71(64-74)$ \\
Body mass index $(\mathrm{kg} / \mathrm{m} 2)$ & $21.9(20.9-24.9)$ & $24.7(23.0-29.0)$ & $29.4(24.8-32.9)$ & $24.8(23.7-26.9)$ & $28.0(24.6-33.3)$ \\
Sublingual temperature $\left({ }^{\circ} \mathrm{C}\right)$ & $36.5(36.3-37.0)$ & $36.4(36.0-36.7)$ & $36.2(36.0-36.5)$ & $36.6(36.2-37.2)$ & $36.5(36.5-36.8)$ \\
Mean arterial pressure $(\mathrm{mmHg})$ & $93(91-96)$ & $100(93-109)$ & $92(88-102)$ & $98(89-106)$ & $98(89-106)$ \\
Heart rate $(\mathrm{bpm})$ & $72(63-82)$ & $75(65-83)$ & $72(62-86)$ & $74(64-92)$ & $70(65-80)$ \\
\hline
\end{tabular}

Data presented as $n$ (\%) or median (IQR). $n=20$ for all groups except cirrhosis where $n=18$. 


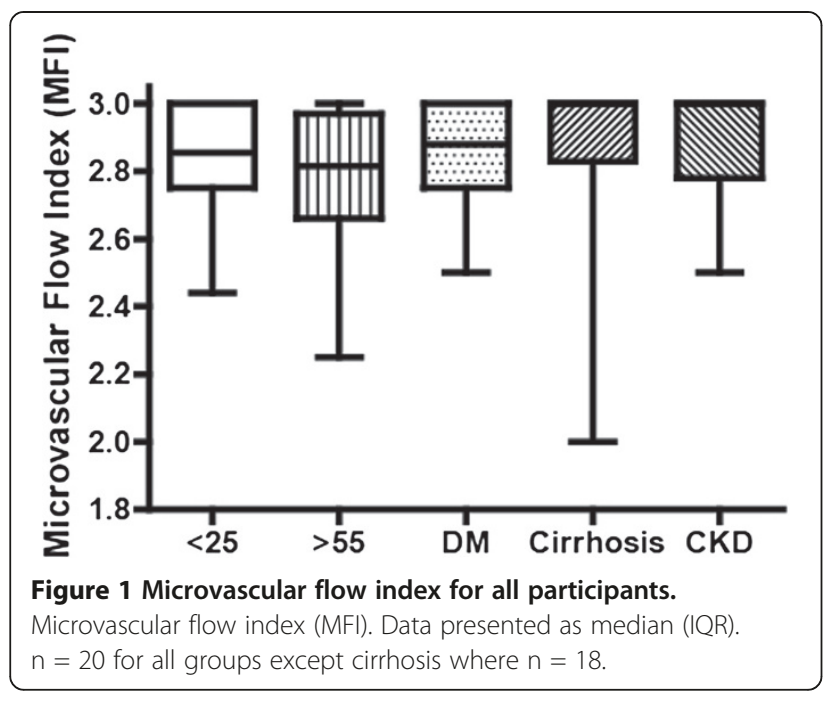

aged $>55$ years, 20 with diabetes, 18 with cirrhosis, and 20 with stage $5 \mathrm{CKD}$ ). Participant characteristics and baseline clinical data are displayed in Table 1 . All the healthy young participants had no medical problems, were non-smokers and declared no regular medication use. Two healthy older participants took medication for hypothyroidism (normal thyroid function tests), two were smokers, two took statins and two took antihypertensive medication, all for primary prevention of cardiovascular disease. All diabetic patients suffered from type 2 diabetes, nine took insulin and 15 took antihypertensive medication. The mean glycated haemoglobin (HbA1c) for patients with diabetes was $8.8 \%$ (SD 1.7\%). For participants with cirrhosis, three took propranolol for variceal bleeding prophylaxis, and eight took other antihypertensive medication. The cause of cirrhosis was viral in 11 cases, alcohol-related in five cases, medication-related in one case and cryptogenic in one case. Seventeen patients were Child-Pugh-Turcotte score A and one was score B. For patients with stage 5 CKD, this was due to intrinsic renal disease in seven cases, polycystic kidney disease in one case, reflux nephropathy in one case, drug toxicity in one case, and hypertension in ten cases. All were taking antihypertensive medication. The mean estimated glomerular filtration rate was $11.5 \mathrm{ml} / \mathrm{min}$ (SD 2.9).

A median of 3 (IQR 2 to 4) clips were analysed for each patient. For small vessels $(<20 \mu \mathrm{m})$, there were no statistically significant differences in MFI, PPV and PVD between the groups (Figure 1 and Table 2). This finding was repeated in a secondary analysis that excluded the two hypertensive participants from the healthy $>55$ group. Baseline sublingual large vessel $(>20 \mu \mathrm{m})$ MFI was median 3.0 (IQR 2.9 to 3.0) and PPV was median 1.0 (IQR 0.97 to 1.0), suggesting good quality image capture unaffected by pressure artefact.

\section{Discussion}

The principal finding of this study was that sublingual SDF imaging does not demonstrate statistically significant differences in MFI, PPV and PVD between healthy young volunteers, healthy older volunteers, and patients with diabetes, cirrhosis and end-stage renal failure. MFI was lower in older volunteers and higher in those with cirrhosis and renal failure, and PVD was higher in patients with diabetes, cirrhosis and renal failure. However, none of these trends reached significance.

There is little doubt that age, diabetes, cirrhosis and renal disease do cause alterations to the microvasculature. Diabetes, particularly type 2 diabetes, is associated with increased capillary density. This has been recently demonstrated using SDF imaging of the labial microcirculation [23]. Our results showed a trend towards an increased PVD that was not statistically significant. Similarly, our results indicated a non-significant trend towards higher PVD and MFI values in cirrhotic and CKD patients. In cirrhosis, vasodilatory mediators promote arteriovenous shunting, eventually leading to the development of a hyperdynamic circulation. Cirrhotic patients show an exaggerated post-ischaemic hyperaemia response, which has been interpreted as evidence that the peripheral microvasculature is predisposed to vasodilatation [24]. Sheikh and colleagues recorded seemingly low MFI values in their comparison of SDF analysis of compensated, decompensated and septic cirrhotic patients [25], but as their study did not include any healthy patients, comparison with our own results is not possible. Using nailfold microscopy, Thang and colleagues found a trend towards reduced capillary density in CKD stage 5 patients compared to controls, although this did not reach significance [26]. Bemelmans and colleagues compared MFI in dialysis patients before and after dialysis [27]. The baseline MFI values they

Table 2 SDF microvascular flow parameters for all participants

\begin{tabular}{lcccccc}
\hline & Age $<\mathbf{2 5}$ years & Age $>\mathbf{5 5}$ years & Diabetes mellitus & Cirrhosis & Chronic kidney disease & $\boldsymbol{P}$ value \\
\hline MFI & $2.85(2.75-3.0)$ & $2.81(2.66-2.97)$ & $2.88(2.75-3.0)$ & $3.0(2.83-3.0)$ & $3.0(2.78-3.0)$ & 0.14 \\
PPV & $0.92(0.06)$ & $0.88(0.09)$ & $0.92(0.07)$ & $0.89(0.08)$ & $0.91(0.05)$ & 0.46 \\
PVD & $8.04(1.49)$ & $8.02(0.96)$ & $9.17(1.70)$ & $8.51(1.43)$ & $8.38(1.36)$ & 0.08 \\
\hline
\end{tabular}

PPV in \%, PVD in $\mathrm{mm}^{-1}$. Data presented as mean (SD) or median (IQR). $n=20$ for all groups except cirrhosis where $n=18$. MFI, microvascular flow index; PPV, proportion of perfused vessels; PVD, perfused vessel density. 
found are similar to those we report, but without a control group in their study, comparison is not possible.

We found that microvascular flow as measured by SDF parameters was not significantly different when subjects were older or had comorbid diabetes, chronic kidney disease or cirrhosis. It is possible that a much larger study than ours may have identified differences between these groups and young, healthy subjects using SDF parameters. It is also possible that alternative techniques may have detected differences that were not apparent using SDF imaging. However, our aim was to identify important confounding effects in the use of SDF parameters to assess for acute changes in the microcirculation of critically ill patients. Assessment of the microcirculation specifically using SDF imaging has generated significant research interest in perioperative medicine and critical care, in part because this technique can be used in vivo with minimal disruption to the subject and could potentially form an important part of bedside clinical evaluation. In calculating our sample size, we specified an effect size equal to a 0.26 difference in MFI, on the basis that effect sizes of this magnitude have been seen in previous studies of critically ill patients. Thus we believe our results show that no such confounding effects need be taken into account.

We cannot rule out the possibility that pre-existing microvascular dysfunction not apparent on SDF imaging may predispose patients with chronic diseases to SDFdetectable microvascular flow alterations when they become acutely unwell. Further research would be needed to investigate the importance of any such effect.

\section{Conclusions}

Advancing age, diabetes mellitus, chronic kidney disease and cirrhosis do not appear to cause changes in sublingual microvascular flow assessed by sidestream dark field imaging in stable patients.

\section{Abbreviations}

CKD: chronic kidney disease; FCD: functional capillary density: IQR: interquartile range; MFI: microvascular flow index; OPS: orthogonal polarisation spectral; PPV: proportion of perfused vessels; PVD: perfused vessel density; SD: standard deviation; SDF: sidestream dark field.

\section{Competing interests}

The authors declare they have no competing interests.

\section{Authors' contributions}

TR, AVS, SJ and RP contributed to the study conception and design. TR and AVS recruited participants, recorded and analysed the data. TR and SJ performed the statistical analysis. TR, AVS, SJ and RP contributed to interpretation of the data and drafting the manuscript. All authors read and approved the final manuscript.

Authors' information

RP is a National Institute for Health Research (UK) Clinician Scientist.

\section{Acknowledgements}

This study was supported with a grant from the Isaac Schapera Trust for Medical Research. The funding body had no role in study design, collection, analysis, or interpretation of data, preparation of the manuscript or in the decision to submit the manuscript for publication.

We thank Professor Magdi Yaqoob, Professor Graham Foster and Dr Tahseen Chowdhury for their assistance in recruiting participants to this study. SJ acknowledges UK National Health Service funding to the Royal Marsden/ Institute of Cancer Research National Institute for Health Research (UK) Biomedical Research Centre.

Elements of this study were presented at the 33rd International Symposium on Intensive Care and Emergency Medicine in abstract form.

\section{Author details}

'Adult Critical Care Unit, Royal London Hospital, Barts Health NHS Trust, Whitechapel Rd, London E1 1BB, UK. ${ }^{2}$ Intensive Care Unit, Royal Marsden Hospital, Fulham Road, London SW3 6JJ, UK. ${ }^{3}$ Barts and The London School of Medicine and Dentistry, Queen Mary's University of London, Turner Street, London E1 2AD, UK.

Received: 9 February 2013 Accepted: 15 March 2013

Published: 9 April 2013

\section{References}

1. Spronk PE, Zandstra DF, Ince C: Bench-to-bedside review: sepsis is a disease of the microcirculation. Crit Care 2004, 8:462-468.

2. De Backer D, Creteur J, Preiser J-C, Dubois M-J, Vincent J-L: Microvascular blood flow is altered in patients with sepsis. Am J Respir Crit Care Med 2002, 166:98-104.

3. Sakr Y, Dubois M-J, De Backer D, Creteur J, Vincent J-L: Persistent microcirculatory alterations are associated with organ failure and death in patients with septic shock. Crit Care Med 2004, 32:1825-1831.

4. Spanos A, Jhanji S, Vivian-Smith A, Harris T, Pearse RM: Early microvascular changes in sepsis and severe sepsis. Shock 2010, 33:387-391.

5. Jhanji S, Lee C, Watson D, Hinds C, Pearse RM: Microvascular flow and tissue oxygenation after major abdominal surgery: association with postoperative complications. Intensive Care Med 2009, 35:671-677.

6. Jhanji S, Vivian-Smith A, Lucena-Amaro S, Watson D, Hinds CJ, Pearse RM: Haemodynamic optimisation improves tissue microvascular flow and oxygenation after major surgery: a randomised controlled trial. Crit Care 2010, 14:R151.

7. Elbers PWG, Ince C: Mechanisms of critical illness - classifying microcirculatory flow abnormalities in distributive shock. Crit Care 2006, 10:221.

8. Elbers PWG, Prins WB, Plokker HWM, van Dongen EPA, van Iterson M, Ince C: Electrical cardioversion for atrial fibrillation improves microvascular flow Independent of blood pressure changes. J Cardiothorac Vasc Anesth 2012, 26:799-80

9. Jhanji S, Stirling S, Patel N, Hinds CJ, Pearse RM: The effect of increasing doses of norepinephrine on tissue oxygenation and microvascular flow in patients with septic shock. Crit Care Med 2009, 37:1961-1966.

10. Dubin A, Pozo MO, Casabella CA, Pálizas F Jr, Murias G, Moseinco MC, Kanoore Edul VS, Pálizas F, Estenssoro E, Ince C: Increasing arterial blood pressure with norepinephrine does not improve microcirculatory blood flow: a prospective study. Crit Care 2009, 13:R92.

11. Bangash M, Pearse RM: Microcirculation. In Encyclopedia of intensive care medicine. Edited by Vincent JL, Hall JB. London: Springer; 2012:1395-1399.

12. Mathura KR, Vollebregt KC, Boer K, De Graaff JC, Ubbink DT, Ince C: Comparison of OPS imaging and conventional capillary microscopy to study the human microcirculation. J Appl Physiol 2001, 91:74-78.

13. Goedhart PT, Khaliizada M, Bezemer R, Merza J, Ince C: Sidestream Dark Field (SDF) imaging: a novel stroboscopic LED ring-based imaging modality for clinical assessment of the microcirculation. Opt Express 2007, 15:15101-15114.

14. Knaus WA, Wagner DP, Draper EA, Zimmerman JE, Bergner M, Bastos PG, Sirio CA, Murphy DJ, Lotring T, Damiano A: The APACHE III prognostic system. Risk prediction of hospital mortality for critically ill hospitalized adults. Chest 1991, 100:1619-1636.

15. Esper AM, Martin GS: The impact of comorbid conditions on critical illness. Crit Care Med 2011, 39:2728-2735. 
16. Poole D, Behnke B, Musch T: Capillary hemodynamics and oxygen pressures in the aging microcirculation. Microcirculation 2006, 13:289-299.

17. Granger DN, Rodrigues SF, Yildirim A, Senchenkova EY: Microvascular responses to cardiovascular risk factors. Microcirculation 2010, 17:192-205.

18. Sherman IA, Pappas SC, Fisher MM: Hepatic microvascular changes associated with development of liver fibrosis and cirrhosis. Am J Physiol 1990, 258:H460-H465.

19. Stewart J, Kohen A, Brouder D, Rahim F, Adler S, Garrick R, Goligorsky MS: Noninvasive interrogation of microvasculature for signs of endothelial dysfunction in patients with chronic renal failure. Am J Physiol Heart Circ Physiol 2004, 287:H2687-H2696.

20. Boerma EC, Mathura KR, van der Voort PHJ, Spronk PE, Ince C: Quantifying bedside-derived imaging of microcirculatory abnormalities in septic patients: a prospective validation study. Crit Care 2005, 9:R601-R606.

21. De Backer D, Hollenberg S, Boerma C, Goedhart P, Büchele G, OspinaTascon G, Dobbe I, Ince C: How to evaluate the microcirculation: report of a round table conference. Crit Care 2007, 11:R101.

22. Levey AS, Coresh J, Greene T, Stevens LA, Zhang YL, Hendriksen S, Kusek JW, Van Lente F, Chronic Kidney Disease Epidemiology Collaboration: Using standardized serum creatinine values in the modification of diet in renal disease study equation for estimating glomerular filtration rate. Ann Intern Med 2006, 145:247-254.

23. Djaberi R, Schuijf JD, de Koning EJ, Wijewickrama DC, Pereira AM, Smit JW, Kroft LJ, de Roos A, Bax JJ, Rabelink TJ, Jukema JW: Non-invasive assessment of microcirculation by sidestream dark field imaging as a marker of coronary artery disease in diabetes. Diab Vasc Dis Res 2013, 10:123-134. Epub 2012 May 23.

24. Thomson SJ, Cowan ML, Forton DM, Clark SJ, Musa S, Grounds M, Rahman TM: A study of muscle tissue oxygenation and peripheral microcirculatory dysfunction in cirrhosis using near infrared spectroscopy. Liver Int 2010, 30:463-471. Epub 2009 Nov 16.

25. Sheikh MY, Javed U, Singh J, Choudhury J, Deen O, Dhah K, Peterson MW: Bedside sublingual video imaging of microcirculation in assessing bacterial infection in cirrhosis. Dig Dis Sci 2009, 54:2706-2711.

26. Thang OH, Serné EH, Grooteman MP, Smulders YM, ter Wee PM, Tangelder GJ, Nubé MJ: Capillary rarefaction in advanced chronic kidney disease is associated with high phosphorus and bicarbonate levels. Nephrol Dial Transplant 2011, 26:3529-3536. Epub 2011 Mar 17.

27. Bemelmans RH, Boerma EC, Barendregt J, Ince C, Rommes JH, Spronk PE: Changes in the volume status of haemodialysis patients are reflected in sublingual microvascular perfusion. Nephrol Dial Transplant 2009, 24:3487-3492

doi:10.1186/2047-0525-2-7

Cite this article as: Reynolds et al:: Observational study of the effects of age, diabetes mellitus, cirrhosis and chronic kidney disease on sublingual microvascular flow. Perioperative Medicine 2013 2:7.

\section{Submit your next manuscript to BioMed Central and take full advantage of:}

- Convenient online submission

- Thorough peer review

- No space constraints or color figure charges

- Immediate publication on acceptance

- Inclusion in PubMed, CAS, Scopus and Google Scholar

- Research which is freely available for redistribution 